\title{
Quality of Life in Patients With Hepatocellular Carcinoma Received Surgical Resection
}

\author{
LUKAS JYUHN-HSIARN LEE, ${ }^{1,2}$ CHIEN-HUNG CHEN, MD, PhD ${ }^{3 *}$ GRACE YAO, ${ }^{4}$ CHIH-WEN CHUNG, ${ }^{2}$ \\ JIN-CHUAN SHEU, ${ }^{3}$ PO-HUANG LEE, ${ }^{5}$ YIH-JIAN TSAI, ${ }^{6}$ AND JUNG-DER WANG ${ }^{2,7}$ \\ ${ }^{1}$ Department of Neurology, National Taiwan University Hospital \\ and National Taiwan University College of Medicine, Taipei, Taiwan \\ ${ }^{2}$ Center for Health Risk Assessment and Policy, College of Public Health, \\ National Taiwan University, Taipei, Taiwan \\ ${ }^{3}$ Department of Internal Medicine, National Taiwan University Hospital \\ and National Taiwan University College of Medicine, Taipei, Taiwan \\ ${ }^{4}$ Department of Psychology, College of Science, National Taiwan University, Taipei, Taiwan \\ ${ }^{5}$ Department of Surgery, National Taiwan University Hospital and \\ National Taiwan University College of Medicine, Taipei, Taiwan \\ ${ }^{6}$ Bureau of Health Promotion, Department of Health, Taipei, Taiwan \\ ${ }^{7}$ Institute of Occupational Medicine and Industrial Hygiene, College of Public Health, \\ National Taiwan University, Taipei, Taiwan
}

\begin{abstract}
Background: Hepatocellular carcinoma (HCC) is one the most common malignancies in the world. As the prognosis for HCC patients is poor, the quality of life (QOL) is becoming more important on the outcome assessments. The aim of this study was to evaluate QOL in HCC patients.

Methods: A total of 161 patients with $\mathrm{HCC}$ were enrolled at a university hospital. Most of these patients received surgical resections. They were assessed with Taiwan versions of WHOQOL-BREF, EORTC QLQ-C30, and utility measures. The WHOQOL-BREF domain scores for the HCC patients were compared to healthy normative Taiwan population, using general linear models controlling for gender and age. Multiple logistic regressions were applied to explore association between a better QOL and clinical/sociodemographic variables.

Results: Compared with healthy people, the HCC patients had reduced QOL in physical domains, but better environmental QOL. After controlling gender, age, education, and employment, duration of HCC more than 1 year was associated with better QOL scores. WHOQOL-BREF could be cross-validated with EORTC QLQ-C30. Conclusions: Survival over 1 year was associated with better QOL in HCC patients. WHOQOL-BREF could be a valid QOL instrument for the assessments of QOL in HCC patients.
\end{abstract}

J. Surg. Oncol. 2007;95:34-39. (c) 2006 Wiley-Liss, Inc.

KEY Words: EORTC QLQ-C30; hepatocellular carcinoma; quality of life; utility; WHOQOL-BREF

\section{INTRODUCTION}

Hepatocellular carcinoma (HCC) is one the most common malignancies in the world, especially in subSaharan Africa and Southeast Asia [1]. Since 1984, it has been the leading cause of cancer death in Taiwan. About $6,000-8,000$ people died of this cancer every year in Taiwan [2]. A trend of increasing incidence of HCC has been disclosed in the Western world, which may be associated with chronic infections of hepatitis viruses
Chien-Hung Chen and Jung-Der Wang contribute equally to this work. Grant sponsor: National Health Research Institutes, Taiwan; Grant number: NHRI-EX92-9204PP.

*Correspondence to: Chien-Hung Chen, MD, PhD, Department of Internal Medicine, National Taiwan University Hospital and National Taiwan University College of Medicine, Taipei, Taiwan, 7, Chung-Shan South Road, Taipei 100, Taiwan. Fax: 886-2-23819723.

E-mail: chenhcc@ntumc.org

Received 12 April 2005; Accepted 3 August 2005

DOI 10.1002/jso.20374

Published online in Wiley InterScience (www.interscience.wiley.com). 
$[3,4]$. As the prognosis for HCC patients is poor, with a 5 -year survival less than $50 \%$ even in resectable patients [5,6] and mean overall survival less than 5 years [7], the quality of life (QOL) is becoming more important on the outcome assessment for clinical services [8].

QOL is an important dimension of health, and it has been defined by the World Health Organization (WHO) as "individual's perceptions of their position in life in the context of the culture and value systems in which they live and in relation to their goals, expectations, standards, and concerns." In 1991, the WHO initiated a project to develop a generic QOL instrument, which led to the WHOQOL assessment $[9,10]$. There has been a rapid growth in the assessment of health-related quality of life (HRQOL) as a technique for clinical research since 1970s [11]. Up to 2000, there were at least one thousand different measurements of HRQOL reviewed by Tengs and Wallace [12], and a comprehensive table was compiled to provide quality weights for many diseases. A large proportion of these QOL weights, however, were based on the viewpoints of authors and experts. Patients' preference was the main concern in about $39 \%$ of all respondents. Empirical studies using patient-centered QOL are warranted for quantifying effectiveness in clinical research [13]. QOL assessments can improve care for cancer patients. Thus, we conducted the study to assess QOL in HCCs patients, focusing on the surgically treated patients.

\section{METHODS}

\section{Subjects}

One hundred sixty one patients with HCC were recruited at the National Taiwan University Hospital (NTUH) from February to April 2002. The diagnosis of HCC was confirmed by histological evidence or clinical diagnosis mainly based on positive findings of image studies and AFP levels higher than $400 \mathrm{ng} / \mathrm{ml}$. The institutional review board approved this study and an informed consent was obtained from every participant prior to the survey. The clinical characteristics of the 161 patients were summarized in Table I. Most of these patients had compensated liver diseases and received surgical resections for their HCCs.

\section{Instruments for QOL Assessment}

The participants were requested to evaluate their QOL over the past 2 weeks with a self-administrated questionnaire in hospital. The instruments for measuring QOL in our study were briefed as follows:

(A) Generic instrument: The WHOQOL-BREF Taiwan version with a 5-point Likert response scale has been developed with good reliability and validity [14]. It
TABLE I. Clinical Characteristics of the 161 HCC Patients

\begin{tabular}{|c|c|}
\hline Characteristics & $\mathrm{N}$ (\% of patients) \\
\hline Gender: male /female & $122(75.8) / 39(24.2)$ \\
\hline \multicolumn{2}{|l|}{ Age (year) } \\
\hline Median, and range & $62,21-90$ \\
\hline Mean \pm SD & $61.6 \pm 12.4$ \\
\hline Education of high school or more & $79(49.1)$ \\
\hline Married & $135(83.9)$ \\
\hline Have current job & $63(39.1)$ \\
\hline \multicolumn{2}{|l|}{ Virology } \\
\hline HBsAg (+) & 98 (63.6) \\
\hline Anti-HCV (+) & $53(42.4)$ \\
\hline \multicolumn{2}{|l|}{ Biochemical values } \\
\hline \multicolumn{2}{|l|}{ Prothrombin time prolonged $(\mathrm{sec})$} \\
\hline$<4 / 4-6 />6$ & $142(88.2) / 15(9.3) / 4(2.5)$ \\
\hline \multicolumn{2}{|l|}{ Albumin (g/dl) } \\
\hline$\geq 3.5 / 2.8-3.4 /<2.8$ & $134(83.3) / 25(15.5) / 2(1.2)$ \\
\hline \multicolumn{2}{|l|}{ Bilirubin $(\mathrm{mg} / \mathrm{dl})$} \\
\hline$<2 / 2-3 />3$ & $157(97.5) / 3(1.9) / 1(0.6)$ \\
\hline \multicolumn{2}{|l|}{ ALT } \\
\hline$<2 \mathrm{ULN} / \geq 2 \mathrm{ULN}$ & $116(72.0) / 45(28.0)$ \\
\hline \multicolumn{2}{|l|}{ Child-Pugh classification } \\
\hline $\mathrm{A} / \mathrm{B} / \mathrm{C}$ & $150(93.1) / 8(5.0) / 3(1.9)$ \\
\hline \multicolumn{2}{|l|}{ Size of tumor $(\mathrm{cm})$} \\
\hline$\leq 3 / 3-5 />5$ & $74(46.0) / 41(25.5) / 46(28.5)$ \\
\hline \multicolumn{2}{|l|}{ Modality of diagnosis } \\
\hline Histopathology & $123(76.4)$ \\
\hline Imaging $+\mathrm{AFP} \geq 400 \mathrm{ng} / \mathrm{ml}$ & $12(7.5)$ \\
\hline Imaging + AFP $<400$ ng/ml & $26(16.1)$ \\
\hline \multicolumn{2}{|l|}{ Cirrhosis } \\
\hline Present & $91(56.5)$ \\
\hline \multicolumn{2}{|l|}{ Duration of disease } \\
\hline$\leq 1$ year $/ 1-3$ years $/ \geq 3$ years & $31(19.2) / 75(46.6) / 55(34.2)$ \\
\hline \multicolumn{2}{|l|}{ Survey subject } \\
\hline Outpatient & $131(81.4)$ \\
\hline \multicolumn{2}{|l|}{ Treatment modalities } \\
\hline Surgical/TACE/PEI/supportive & $121(75.2) / 31(19.2) / 8(5.0) / 1(0.6)$ \\
\hline
\end{tabular}

HBsAg, hepatitis B surface antigen; anti-HCV, antibody for hepatitis $\mathrm{C}$ virus; ULN, upper limit of normal; TACE, transcatheter arterial chemoembolization; PEI, percutaneous ethanol injection.

contains the 26 original items of the WHOQOL-BREF, plus two additional items of national importance. The first two questions concern overall QOL and general health, and the remaining 24 items can be grouped into four domains as follows: physical, psychological, social, and environment. Two national items, "being respected/accepted" and "eating/food" were included in the Taiwan version, each from a culture-specific facet that constitutes social (TW), and environment (TW) domains, respectively.

(B) Cancer-specific instrument: The European Organization for Research and Treatment of Cancer Quality of Life Questionnaire Core-30 (EORTC QLQ-C30, version 3.0) is a cancer-specific QOL instrument originally designed for use in cancer clinical trials. The questionnaire includes five functional scales (physical, role, social, emotional, and cognitive), global QOL/general 
health, and symptoms frequently reported by cancer patients (fatigue, nausea/vomiting, pain, dyspnea, insomnia, appetite loss, constipation, diarrhea) as well as an item on the financial impact [15]. It consists of 30 items, uses a 4-point scale for 28 items and a 7-point scale for two overall measures. The Taiwanese version of QLQ$\mathrm{C} 30$ has been developed through formal processes and field test and verified [16].

(C) Utility: Utility was measured with the visualanalogue scale (VAS) and standard gamble (SG) method. To assess utility by means of VAS, the participants were asked to rate their overall health-related QOL in the recent two weeks from 0 ("worst" condition) to 100 ("best" condition). With a flow diagram of making choices of taking a hypothetical curative therapy in the SG, the quality weight of HCC was assessed by comparing his/her health state to a gamble with a probability $(P)$ of achieving full health and a complementary probability $(1-P)$ of death. The probability of full health was titrated from $99 \%$ down to $5 \%$ until the HCC patient was indifferent between the alternatives, and the quality weight of this HCC patient would be equal to the probability $P$ [17].

\section{Comparison With Healthy Population}

For comparison purposes, a group of healthy control subjects (the healthy population, $\mathrm{n}=8,922$ ) was taken from the National Health Interview Survey in 2001, a representative national sample of noninstitutionalized adults in Taiwan (http://nhis.nhri.org.tw/). The healthy population was selected by excluding subjects who reported chronic diseases diagnosed by doctors including cardiac disease, pulmonary disease, hypertension, diabetic mellitus, stroke, peptic ulcer, sinusitis, liver disease, renal disease, and blindness. Their QOL was measured with the WHOQOL-BREF Taiwan version. All QOL scores were transformed by means of linear transformation into a $0-100$ metric, with higher scores indicating better health [18]. Since the patients in this study were significantly older and included a higher proportion of males, the domain scores of the WHOQOL-BREF were compared between the HCC patients and the healthy population using the general linear model approach controlling for age and gender [19].

\section{Relation Between QOL and Variables}

We carried out multivariable logistic regression analysis to examine the relative impacts of the sociodemographic characteristics and clinical variables on the QOL scores that the upper half was defined as better QOL compared with the lower half. We treated potential determinants as dichotomous variables that were significant in univariate analyses, including gender (male vs. female), age ( $\geq 50$ vs. $<50$ years), education (high school or more vs. less than high school), and duration of disease $(>1$ vs. $\leq 1$ years) and treatment (surgery vs. non-surgical treatment). Data analyses were performed using SAS (Statistical Analysis System) software Version 8.2. Twotailed $P$ values of less than 0.05 were considered to be statistically significant.

\section{RESULTS}

\section{Internal Consistency Reliability}

The four domain scores of WHOQOL-BREF were significantly correlated with overall QOL (ranging from 0.36 for physical domain to 0.48 for psychological domain, all $P<0.0001$ ) and general health (ranging from 0.25 for social domain with $P<0.005-0.45$ for physical domain with $P<0.0001$ ). In addition, the domain scores were all moderately correlated with utility measures using VAS (ranging from 0.40 for social domain to 0.59 for physical domain, all $P<0.0001$ ), also to a lesser extent with SG utility (ranging from 0.23 for social domain with $P<0.005$ to 0.38 for psychological domain with $P<0.0001)$. Significant item-domain correlations (all $P<0.0001$ ) ranged from 0.56 for pain to 0.84 for activities of daily living (physical domain), from 0.53 for negative feeling to 0.75 for self-esteem (psychological domain), from 0.75 for sexual life to 0.79 for social support (social domain), from 0.52 for social care to 0.68 for leisure activity (environment domain).

The Cronbach's alpha coefficients for the four domains of WHOQOL-BREF ranged from 0.65 in social domain to 0.83 in physical domain. It suggested that items within domains of WHOQOL-BREF were moderately internally consistent. The internal consistency for the entire WHOQOL-BREF and EORTC QLQ-C30 instruments was adequate with an alpha of 0.91 and 0.84 , respectively.

\section{Correlations Between the Corresponding Subscales of WHOQOL-BREF and EORTC QLQ-C30}

Correlations between the corresponding subscales of WHOQOL-BREF and EORTC QLQ-C30 ranged from $r=0.17$ for the social domain (poor agreement) to $r=0.68$ for the physical domain (good agreement). Some symptom subscales of EORTC QLQ-C30, including pain, fatigue, insomnia, and financial difficulties, were significantly correlated with the corresponding items of WHOQOLBREF, ranging from $r=0.48$ for pain to $r=0.77$ for insomnia (all $P<0.0001$ ), indicating good convergent validity for these two measurement tools. Exploratory factor analysis revealed similar four factors (physical, psychosocial, resource environment, and physical environment) that corresponded to the structure of WHOQOLBREF domains. 
TABLE II. Summary Scores of the Four Domains of WHOQOL-BREF: Comparisons of the HCC Patients With Taiwanese Healthy Population

\begin{tabular}{|c|c|c|c|c|c|c|c|}
\hline \multirow[b]{2}{*}{ WHOQOL-BREF } & \multicolumn{3}{|c|}{ HCC patients } & \multicolumn{3}{|c|}{ Healthy population } & \multirow[b]{2}{*}{ General linear model $P$ value } \\
\hline & $\mathrm{N}$ & Mean & SD & $\mathrm{N}$ & Mean & SD & \\
\hline Physical & 161 & 59.1 & 17.8 & 8,911 & 70.7 & 12.1 & $<0.0001$ \\
\hline Psychological & 161 & 58.8 & 14.0 & 8,913 & 61.3 & 13.7 & 0.07 \\
\hline Social $^{\mathrm{a}}$ & 161 & 63.0 & 13.5 & 8,909 & 65.7 & 13.4 & 0.33 \\
\hline Social $(\mathrm{TW})^{\mathrm{b}}$ & 161 & 62.7 & 13.1 & 8,906 & 63.8 & 12.8 & 0.85 \\
\hline Environment $\mathrm{t}^{\mathrm{a}}$ & 161 & 62.4 & 12.7 & 8,919 & 57.0 & 13.2 & $<0.0001$ \\
\hline Environment $(\mathrm{TW})^{\mathrm{b}}$ & 161 & 63.8 & 12.6 & 8,919 & 58.3 & 12.8 & $<0.0001$ \\
\hline
\end{tabular}

All scores were transformed to a $0-100$ metric, using linear transformation. $P$ values are shown for differences between HCC patients and the healthy population in each domain score of WHOQOL-BREF using the general linear models controlling for gender and age (employment).

${ }^{a}$ Domain excluding culture-specific items.

${ }^{\mathrm{b}}$ Domain including culture-specific items.

\section{Impact of HCC on QOL Compared to Healthy Controls}

Table II shows comparison of mean QOL scores between the HCC patients and the healthy population. The $t$-tests revealed the patients with HCC had significantly lower QOL in physical, psychological, and social domains, but higher QOL in environmental domain. After adjustment for gender and age using general linear models, statistically significant differences were found in the physical and environment domains. The HCC patients had lower scores in the physical domain, while they had higher scores in the environment domain.

\section{Factors Associated With QOL in HCC Patients}

The surgery group seemed to have a significantly better QOL (data not shown). Using multiple logistic regression analyses to analyze factors affecting QOL, the duration of disease was related to the overall OQL of WHOQOLBREF, global health of EORTC QLQ-C30, and standard gamble (Table III). The other factors did not have important impacts on the QOL of HCC patients.

\section{DISCUSSION}

It was usually anticipated that the QOL would be lower in the cancer patients. However, it was interesting to find the scores on the facets of freedom/physical safety/ security and physical environment (pollution/noise/traffic/climate) of the environmental domain were higher in the HCC patients. The reasons for such paradoxical results were not very clear. It is possible that the HCC patients who successfully received surgical treatment subjectively cherish the surrounding environment more than healthy people, as they were put into a situation directly facing the uncertainty on life and death before surgery.

In addition to the WHOQOL-BREF, the other generic HRQOL used in this study was utility. Utility measured using the SG method can be linked to a theoretical foundation in the von Neumann-Morgenstern expected utility theory, and therefore is the preferred estimate for cost-effectiveness analysis under condition of uncertainties [19]. There were similar proportions of an extreme value of SG utility between those aged 65 or more $(36.8 \%)$ and the younger $(36.3 \%)$, those educated of high school or more $(35.4 \%)$ and the less educated (37.5\%). Thus, it was unlikely that age or education would significantly bias utility measurement in our study. After multiple logistic regression analyses, only duration of disease had significant influence on SG utility.

It was believed that the EORTC QLQ-C30 was more sensitive to detect cancer-related symptoms, and therefore it may be more responsive to clinically important changes and more useful in assessing HRQOL in the

TABLE III. Clinical and Sociodemographic Factors Affecting QOL Scores in HCC Patients Using Multiple Logistic Regression Analyses

\begin{tabular}{lcccc}
\hline & \multicolumn{3}{c}{ OR (95\% CI) } \\
\cline { 2 - 5 } Factors & $\begin{array}{c}\text { WHOQOL-BREF } \\
\text { overall QOL }\end{array}$ & $\begin{array}{c}\text { EORTC QLQ-C30 } \\
\text { global health/QOL }\end{array}$ & $\begin{array}{c}\text { Standard } \\
\text { gamble }\end{array}$ & $\begin{array}{c}\text { Visual analogue } \\
\text { scale }\end{array}$ \\
\hline Duration of disease $(>1$ year vs. $\leq 1$ year) & $2.89(1.10-7.60)$ & $3.51(1.41-8.73)$ & $3.61(1.46-8.91)$ & $0.80(0.28-2.27)$ \\
Gender (male vs. female) & $0.83(0.36-1.92)$ & $1.44(0.61-3.41)$ & $1.20(0.48-3.00)$ & $1.40(0.57-3.44)$ \\
Age $(\geq 50$ year vs. $<50$ year) & $0.49(0.19-1.28)$ & $2.11(0.76-5.85)$ & $1.08(0.39-2.97)$ & $0.59(0.16-2.14)$ \\
Education (high school vs. $<$ high school) & $1.64(0.80-3.37)$ & $1.65(0.78-3.50)$ & $0.63(0.29-1.37)$ & $2.88(1.23-6.73)$ \\
Employment (employed vs. unemployed) & $1.09(0.50-2.37)$ & $2.16(0.91-5.14)$ & $1.38(0.60-3.18)$ & $1.31(0.53-3.27)$ \\
\hline
\end{tabular}


clinical trial setting. This study also demonstrated some discriminating power in EORTC QLQ-C30 for specific symptoms, including constipation and appetite loss, which was not included in WHOQOL-BREF. However, the drawback was that EORTC QLQ-30 may not allow comparison among different health conditions other than cancer. In this regard, the generic instrument, WHOQOLBREF, could be used as complementary to EORTC QLQ30. Through cross-validation with EORTC QLQ-C30, we found that WHOQOL-BREF can be a valid QOL instrument with some items to differentiate the QOL in HCC patients. Cross-cultural comparison of various diseases worldwide may be a potential research topic using WHOQOL-BREF instruments [18]. Because different cancers have different etiologies, epidemiology, clinical problems, and treatments, EORTC group has developed supplement modules for separate cancers, such as pancreatic cancer [20] and colorectal cancer with liver metastases [21]. The EORTC QLQ-C30 and a questionnaire module-EORTC QLQ-HCC18 has recently applied to assess the QOL in HCC patients [22]. However, the drawback was that EORTC QLQ-30 may not allow comparison among different diseases other than cancer. In this regard, the generic instrument, WHOQOLBREF, could be used as complementary to EORTC QLQ30. Through cross-validation with EORTC QLQ-C30, we found that WHOQOL-BREF can be a valid QOL instrument with some items to differentiate the QOL in HCC patients receiving surgery versus non-surgical treatment, meanwhile also allowing a wider range of diseases and conditions to be compared. Cross-cultural comparison of various diseases worldwide may be a potential research topic using WHOQOL-BREF instruments [18]. As the logistic regression analysis (Table III) did not show a statistically significant difference between EORTC QLQ-C30 and WHOQOL-BREF, future studies of QOL may consider adding HCC-specific module to probably improve the responsiveness.

Different clinical and sociodemographic factors may have different impacts on the WHOQOL-BREF, EORTC QLQ-C30 scores, and utility measures. It is not surprising to find that higher QOL scores were detected in the surgical patients because patients who underwent surgery had a much better prognosis. However, our study did not randomly assign these treatments so the differences could not be attributed to different treatments because of potential selection bias. Second, the overwhelming majority of patients were surgically treated, leaving relatively little confidence in the numbers provided for the other groups. Further study was needed to verify that the surgical patients had better QOL compared with other treatment groups.

In this study, we also found that the most powerful factor was duration of disease. HCC patients with HCC diagnosed more than 1 year had higher QOL scores, which were constantly demonstrated in both instruments of WHOQOL-BREF and EORTC QLQ-C30. The longer duration of disease might actually represent the better prognostic patients group. Thus, better QOL was detected. It has been shown that the overall management strategy of HCC in the elderly should not be different from that in younger patients [23]. Our study found that age was not a factor related to QOL. Thus, in term of QOL, our result also support the strategy that the elderly HCC patients should be treated as the younger patients.

Another caution should be taken in interpreting our results because the available respondents may tend to over-estimate QOL of HCC. These 161 participants comprised a consecutive sample of all HCC patients at the NTUH because a random sampling was not practical due to budget constraints and patient availability. Since these patients were recruited consecutively during the 2month study period, they were a quasi-systematic probability sample from the HCC cohort assuming that most patients were eligible for QOL measurement. In fact, $81 \%$ of the respondents were collected at the outpatient clinics and they were very responsive to our survey. HCC patients currently with decompensated hepatic failure, hepatic encephalopathy, under hospice care in the terminal stage, or those suffering from other severe medical complications were hardly feasible for QOL measurement. Moreover, about three-fourths of our patients received surgery, which by itself is a determinant for better QOL. Thus, our QOL data were likely to represent HCC patients who were more stable and better QOL measures, or surgically resectable. Our results might not be generalized to non-surgical HCC patients.

However, our results could be the basis for further studies of OQL in HCCs. It deserves to do both the crosssection and longitudinal studies to further clarify the QOL in HCC patients in the future. In the cross section study, we can compare the QOL in HCC patients received different treatment modalities, such as operation, TAE, local treatment, chemotherapy, or just supportive care. In the longitudinal study, we can compare the QOL in patients before and after the treatments.

In conclusion, we empirically examined the validity of the generic QOL instrument of WHOQOL-BREF in HCC patients through cross-validation with a cancer-specific instrument, EORTC QLQ-C30, and utility measures. The WHOQOL-BREF, in its Taiwan national version, can be a useful generic QOL instrument in HCC patients. The QOL measures of HCC patients were associated with duration of disease. QOL is a crucial clinical endpoint in HCC patients and can be measured with QOL questionnaires in order to provide useful information for clinical management. 


\section{ACKNOWLEDGMENTS}

We are indebted to our colleagues in the Department of Medical Records for their excellent work in cancer registry system. We thank Ms. Li-Chen Chen and HuiYun $\mathrm{Lu}$ for their assistance in patients' interview and chart review.

\section{REFERENCES}

1. Parkin DM, Whelan SL, Ferlay J, et al.: Cancer incidence in five continents. Lyon: IARC Scientific publications, 1997.

2. Chen DS: Hepatitis B virus infection, its sequelae, and prevention in Taiwan. In: Okuda K, Ishak KG, editors. Neoplasms of the liver. Tokyo: Springer-Verlag; 1987. pp 71-80.

3. El-Serag HB, Mason AC: Rising incidence of hepatocellular carcinoma in the United States. N Engl J Med 1999;340:745-750.

4. Bosch FX, Ribes J, Diaz M, et al.: Primary liver cancer: Worldwide incidence and trends. Gastroenterology 2004;127:S5-S16.

5. Huang $\mathrm{YH}$, Chen $\mathrm{CH}$, Chang TT, et al.: The role of transcatheter arterial embolization in patients with resectable hepatocellular carcinoma: A nation-wide, multicenter study. Liver Int 2004; 24:419-424.

6. Song TJ, Wai Kit Ip E, Fong Y: Hepatocellular carcinoma: Current surgical management. Gastroenterology 2004;127:S248S260.

7. Lee WC, Jeng LB, Chen MF: Hepatectomy for hepatitis B-, hepatitis C-, and dual hepatitis B- and C-related hepatocellular carcinoma in Taiwan. J Hepatobiliary Pancreat Surg 2000;7:265-269.

8. Detmar SB, Muller MJ, Schornagel JH, et al.: Health-related quality-of-life assessments and patient-physician communication: A randomized controlled trial. JAMA 2002;288:3027-3034.

9. The World Health Organization Quality of Life Assessment (WHOQOL): Development and general psychometric properties. Soc Sci Med 1998;46:1569-1585.

10. Development of the World Health Organization WHOQOLBREF Quality of Life Assessment: The WHOQOL Group. Psychol Med 1998;28:551-558.
11. Testa MA, Simonson DC: Assessment of quality-of-life outcomes. N Engl J Med 1996;334:835-840.

12. Tengs TO, Wallace A: One thousand health-related quality-of-life estimates. Med Care 2000;38:583-637.

13. Carr AJ, Higginson IJ: Are quality of life measures patient centred? BMJ 2001;322:1357-1360.

14. Yao G, Chung CW, Yu CF, et al.: Development and verification of validity and reliability of the WHOQOL-BREF Taiwan version. J Formos Med Assoc 2002;101:342-351.

15. Aaronson NK, Ahmedzai S, Bergman B, et al.: The European Organization for Research and Treatment of Cancer QLQ-C30: A quality-of-life instrument for use in international clinical trials in oncology. J Natl Cancer Inst 1993;85:365-376.

16. Chie WC, Yang CH, Hsu C, et al.: Quality of life of lung cancer patients: Validation of the Taiwan Chinese version of the EORTC QLQ-C30 and QLQ-LC13. Qual Life Res 2004;13:257-262.

17. Gold MR, Siegel JE, Russel LB, et al.: Cost-effectiveness in health and medicine. New York: Oxford University Press, 1996.

18. World Health Organization: WHOQOL-BREF: Introduction, administration, scoring, and generic version of the assessmentField trial version (available at: http://www.who.int/mental_health/media/en/76.pdf), Geneva, 1996.

19. Littell RC, Freund RJ, Spector PC: SAS system for linear models Cary, NC: SAS Institute; 1991.

20. Fitzsimmons D, Johnson CD, George S, et al.: Development of a disease specific quality of life (QoL) questionnaire module to supplement the EORTC core cancer QoL questionnaire, the QLQC30 in patients with pancreatic cancer. EORTC Study Group on Quality of Life. Eur J Cancer 1999;35:939-941.

21. Kavadas V, Blazeby JM, Conroy T, et al.: Development of an EORTC disease-specific quality of life questionnaire for use in patients with liver metastases from colorectal cancer. Eur J Cancer 2003;39:1259-1263.

22. Blazeby JM, Currie E, Zee BC, et al.: Development of a questionnaire module to supplement the EORTC QLQ-C30 to assess quality of life in patients with hepatocellular carcinoma, the EORTC QLQ-HCC18. Eur J Cancer 2004;40:2439-2444.

23. Poon RT, Fan ST, Lo CM, et al.: Hepatocellular carcinoma in the elderly: Results of surgical and nonsurgical management. Am J Gastroenterol 1999;94:2460-2466. 